\title{
ANÁLISE DE UM SISTEMA DE AQUECIMENTO DE ÁGUA PARA RESIDÊNCIAS RURAIS, UTILIZANDO ENERGIA SOLAR
}

\author{
LUIZ H. BASSO ${ }^{1}$, SAMUEL N. M. DE SOUZA ${ }^{2}$, JAIR A. C. SIQUEIRA ${ }^{3}$, \\ CARLOS E. C. NOGUEIRA ${ }^{4}$, REGINALDO F. SANTOS ${ }^{5}$
}

\begin{abstract}
RESUMO: A conscientização da importância do meio ambiente tem incentivado o estudo de novas fontes energéticas renováveis e menos poluentes. Dentre essas fontes, a energia solar destaca-se por ser perene e limpa. A utilização da energia solar em sistemas de aquecimento de água residencial rural pode colaborar com a economia de energia elétrica, base da matriz energética brasileira. Conhecer os fatores que influenciam na operação de um sistema de aquecimento de água por energia solar é importante na determinação de sua viabilidade técnica, visando a sua difusão em residências rurais. Para tanto, construiu-se um protótipo, no câmpus da Faculdade Assis Gurgacz, em Cascavel - PR, com características similares a um equipamento utilizado em residências para dois habitantes, para funcionar com circulação natural ou termossifão e sem auxílio de sistema de aquecimento complementar. $\mathrm{O}$ equipamento mostrou-se viável tecnicamente, alcançando a temperatura mínima para banho de $35^{\circ} \mathrm{C}$, sempre que a radiação solar foi superior a $3.500 \mathrm{Wh} \mathrm{m}^{-2}$, o que aconteceu para a maioria dos dias estudados.
\end{abstract}

PALAVRAS-CHAVE: água quente, circulação natural, termossolar.

\section{A WATER HEATING SYSTEM ANALYSIS FOR RURAL RESIDENCES, USING SOLAR ENERGY}

\begin{abstract}
The awareness of the importance of the environment has stimulated the study of new renewed energy sources and less pollutant. Amongst these sources, solar energy stands alone for being perennial and clean. The use of solar energy in systems of agricultural residential water heating, can complement the economy of electric energy, base of the Brazilian energy matrix. Knowing the factors that influence the operation of a system of water heating by solar energy is important in determining their technical viabilities targeting their distribution in agricultural residences. To evaluate equipment of water heating for solar energy, a prototype was constructed in the campus of Assis Gurgacz College, in Cascavel,State of Paraná, Brazil, with similar characteristics to equipment used in residences for two inhabitants, to function with natural circulation or thermosiphon and without help of a complementary heating system. The equipment revealed technical viability, reaching the minimum temperature for shower, of $35^{\circ} \mathrm{C}$, whenever the solar radiation was above the $3,500 \mathrm{Wh} \mathrm{m}^{-2}$, for the majority of the studied days.
\end{abstract}

KEYWORDS: hot water, natural circulation, thermosolar.

\footnotetext{
${ }^{1}$ Mestre do Programa de Pós-Graduação em Engenharia Agrícola, CCET-UNIOESTE, Cascavel - PR, melegsouza@ yahoo.com

${ }^{2}$ Prof. Dr. do Mestrado em Energia na Agricultura, CCET-UNIOESTE, Cascavel - PR, ssouza@ unioeste.br

${ }^{3}$ Prof. Dr. do Mestrado em Energia na Agricultura, CCET-UNIOESTE, Cascavel - PR, jairsiqueira@ unioeste.br

${ }^{4}$ Prof. Dr. do Mestrado em Energia na Agricultura, CCET-UNIOESTE, Cascavel - PR, cecn1@ yahoo.com.br

${ }^{5}$ Prof. Dr. do Mestrado em Energia na Agricultura, CCET-UNIOESTE, Cascavel - PR, rfsantos@ unioeste.br

Recebido pelo Conselho Editorial em: 11-9-2008
}

Aprovado pelo Conselho Editorial em: 21-11-2009 


\section{INTRODUÇÃO}

A energia elétrica encontra-se em destaque em todos os setores da economia mundial e é um importante insumo básico para o desenvolvimento do Brasil, que tem na hidroeletricidade sua principal fonte energética. Por outro lado, segundo KOLLING et al. (2004), muitas propriedades e comunidades rurais no Brasil, principalmente nas regiões Norte e Nordeste, não são atendidas com energia elétrica.

Uma maneira de reduzir o consumo de energia elétrica e proporcionar o conforto onde ela não existe, é pela adoção de sistema de aquecimento de água por energia solar. BRAZIL (2006) explica que a grande virtude do aquecimento de água por energia solar é a redução do consumo de energia nas residências no horário de pico, entre 19 e 21 horas, quando há grande aumento de demanda de energia, em decorrência do hábito das pessoas de tomarem banho com chuveiro elétrico.

O sistema de aquecimento residencial de água por energia solar mais indicado é por circulação natural ou termossifão, pela sua simplicidade, confiabilidade e necessitar de pouca manutenção. A circulação ocorre devido à diferença de densidade entre a água fria e a água quente, por convecção. Suas vantagens são a de não consumir energia elétrica, não causar poluição e proporcionar pouca manutenção, que está restrita à limpeza dos vidros.

Seu dimensionamento criterioso é decisivo para sua qualidade. Um bom equipamento, instalado corretamente, mas subdimensionado para as necessidades reais de utilização de água quente, poderá trazer diversos transtornos ao usuário. Um equipamento superdimensionado poderá não trazer o retorno de investimento nos prazos aceitáveis. DHARUMAN et al. (2006) afirmaram que, nas residências, para banho, lavanderia e limpezas, a temperatura requerida da água é de $60{ }^{\circ} \mathrm{C}$ e o consumo de $50 \mathrm{~L}_{\text {pessoa }}{ }^{-1} \mathrm{dia}^{-1}$.

De acordo com as prescrições técnicas de CREDER (2006), para uma residência com duas pessoas, a área de coletores do sistema de aquecimento de água por energia solar necessária é:

$$
\mathrm{S}=\mathrm{Q} \mathrm{I}^{-1} \eta^{-1}
$$

em que,

$\mathrm{S}$ - área em $\mathrm{m}^{2}$

$\mathrm{I}$ - intensidade de radiação solar em $\mathrm{kWh}\left(\mathrm{m}^{2} \mathrm{dia}\right)^{-1}$ ou kcalh $\left(\mathrm{m}^{2} \text { dia }\right)^{-1} \rightarrow$ Adotado como $3.500 \mathrm{Wh} \mathrm{m}^{-2}$ ou $3.011,75 \mathrm{kcal} \mathrm{m}^{-2}$, e

$\eta$ - rendimento do aproveitamento da energia solar, estimado para fins práticos em $50 \%$. Adotado 56,9\%, que foi o rendimento do coletor do protótipo

em que,

$$
\mathrm{Q}=\mathrm{mc} \Delta \mathrm{T}
$$

$\mathrm{m}$ - massa de água $\rightarrow \mathrm{m}=100$ quilos;

$\mathrm{c}$ - calor específico da água $\rightarrow \mathrm{c}=1 \mathrm{kcal} \mathrm{kg}^{-1}{ }^{\circ} \mathrm{C}^{-1}, \mathrm{e}$

$\Delta \mathrm{T}$ - variação de temperatura $\rightarrow \Delta \mathrm{T}=60{ }^{\circ} \mathrm{C}-25^{\circ} \mathrm{C} \rightarrow \Delta \mathrm{T}=35^{\circ} \mathrm{C}$.

Portanto: $\mathrm{Q}=3.500$ kcal e $\mathrm{S}=2,04 \mathrm{~m}^{2}$.

Um coletor de dimensões comerciais de 1,00 x 1,71 m poderá ser adotado conforme as variáveis intensidade de radiação e rendimento do aproveitamento da energia solar [eqs.(1) e (2)]. Isso pode ser confirmado por KULKARNI et al. (2007), que observaram, na Índia, num sistema típico doméstico de aquecimento de água por energia solar, com um coletor de $2 \mathrm{~m}^{2}$, o suprimento diário de 125 litros de água quente com a temperatura da água na ordem de $60{ }^{\circ} \mathrm{C}$.

Tendo em vista o exposto, o presente trabalho teve por objetivo analisar a viabilidade técnica de um sistema de aquecimento de água para residências rurais com dois habitantes, com a utilização de energia solar. 


\section{MATERIAL E MÉTODOS}

Um protótipo de sistema de aquecimento de água por energia solar foi montado no Câmpus da FAG (Faculdade Assis Gurgacz), na Cidade de Cascavel - PR , que está localizada na latitude de 245' sul e longitude de 5330' oeste, com altitude $630 \mathrm{~m}$ e clima subtropical Cfa.

O protótipo foi instalado próximo à estação meteorológica, onde não há possibilidade de sombreamento no coletor. Optou-se por instalações hidráulicas onde a circulação de água entre o coletor solar e o reservatório térmico acontece por termossifão, porque, dessa forma, não há necessidade de usar medidores de vazão de alto investimento e porque é o sistema mais popular e de maior aplicação no Brasil.

Na Figura 1, observa-se o protótipo instalado.
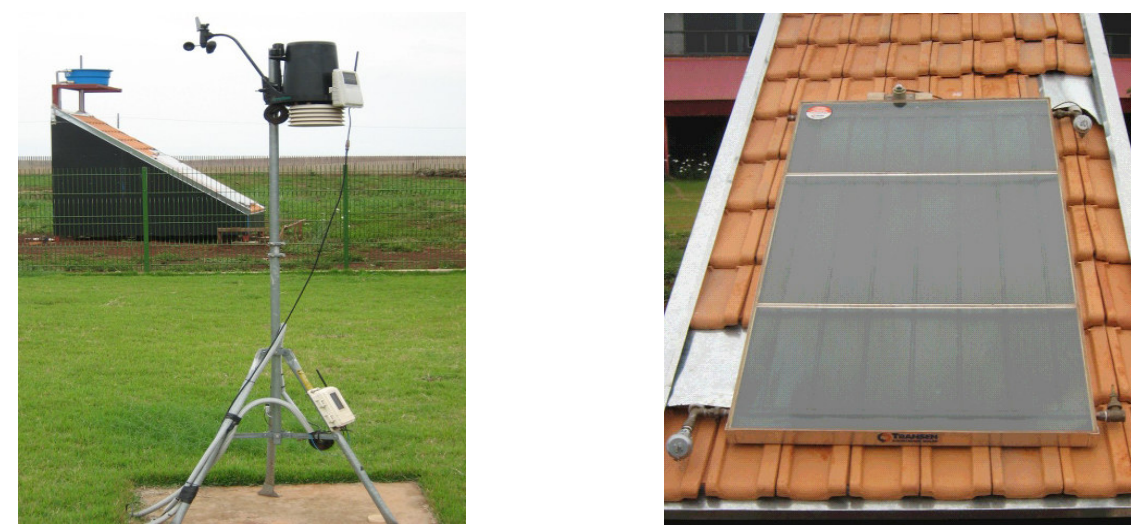

FIGURA 1. À esquerda: vista lateral do protótipo - posição da fotografia: leste - oeste. No primeiro plano, a estação meteorológica. À direita: vista frontal do coletor posição da fotografia: norte - sul. Lados esquerdo inferior e direito superior, os sensores de temperatura. To the left: lateral sight of the prototype - position of the photograph: East - West. In the first plan the meteorological station. To the right: frontal sight of the collector - position of the photograph: NorthSouth. Left inferior and right superior sides, the temperature sensors.

Para a orientação do protótipo, marcou-se o norte magnético em três locais distintos, com auxílio de uma bússola, e criou-se uma linha auxiliar. Dessa, novamente com utilização da bússola, marcou-se $10^{\circ}$ para a direita, ou seja, declinação de $10^{\circ}$ para o nordeste, obtendo-se o norte geográfico. Com esse alinhamento, fez-se um gabarito em madeira e fixou-se o protótipo, parafusando-o com chumbadores metálicos, concretados ao solo. O protótipo operou de forma passiva, por termossifão e em conformidade com as recomendações do fabricante quanto aos materiais utilizados, isolamento térmico, inclinação das tubulações, níveis do reservatório de água fria e de água quente, e orientação e inclinação do coletor solar.

No período de 22 de julho a 16 de setembro de 2007 (inverno), coletaram-se as temperaturas de forma contínua e sem drenagem do boiler, para que fosse possível avaliar sua capacidade de retenção térmica, das $19 \mathrm{~h} 30$ de um dia, às $6 \mathrm{~h}$ do dia seguinte. Por opção, para verificar apenas a parcela de contribuição da energia solar, foi mantido desligado o sistema de aquecimento auxiliar elétrico. Foram medidas as temperaturas ambiente da água do reservatório elevado, na entrada e na saída do coletor solar, no ponto médio do depósito de água quente e no ponto de consumo, que simulou o chuveiro.

Paralelamente, foram adquiridos dados climáticos de temperatura ambiente, velocidade do vento e radiação solar na Estação Meteorológica. Da mesma forma, KHALIFA (1999) utilizou um protótipo para estudar um sistema por circulação natural e para a obtenção da radiação solar e 
temperatura ambiente; serviu-se de dados de uma estação meteorológica automática próxima ao experimento. Especificamente para o caso da radiação solar, como o piranômetro da estação está localizado na horizontal, foi necessário calcular a componente da radiação para uma inclinação do coletor de $35^{\circ}$.

A única manutenção realizada no protótipo foi a limpeza do vidro do coletor, uma vez por mês, operação que demandou em torno de cinco minutos por limpeza.

A avaliação técnica dos dados foi feita em conformidade com a afirmação de CHANG et al. (2004), de que, usualmente, se avaliam sistemas de aquecimento de água funcionando por termossifão, considerando-se o desempenho durante a fase de coleta de energia e a perda térmica na fase de armazenagem de energia. Portanto, foram avaliadas as temperaturas da água, a influência da velocidade do vento no boiler e na placa, a influência da irradiação na temperatura final da água no boiler e o rendimento do boiler.

\section{RESULTADOS E DISCUSSÃO}

Nas Tabelas 1; 2 e 3, são apresentadas as temperaturas coletadas, a velocidade média do vento, a temperatura ambiente média à noite e a temperatura ambiente média ao dia, o cálculo da perda térmica à noite, a perda térmica percentual, a eficiência do boiler na manutenção da energia calorífica e a radiação solar diária.

Pela análise das Tabelas 1; 2 e 3, depreende-se que, mesmo quando a temperatura média durante a noite foi baixa, como, por exemplo, $3,09{ }^{\circ} \mathrm{C}$, na noite de 26-7-2007, a eficiência foi de $81,62 \%$, próxima à da média do período estudado. MADHLOPA et al. (2006) encontraram eficiência média na retenção em torno de $35 \%$. Essa baixa eficiência na retenção, comparada com a alcançada no experimento, deve-se à utilização, pelos autores, de equipamento integrado placa-boiler, em que o boiler fica exposto às intempéries.

A temperatura média alcançada no inverno, ao final do dia, ficou em $57,42{ }^{\circ} \mathrm{C}$. A eficiência e as temperaturas alcançadas demonstram que o dimensionamento sugerido por CREDER (2006) está correto.

TABELA 1. Temperaturas inicial e final no boiler, velocidade do vento, temperatura média ambiente à noite e de dia, perda de energia calorífica e percentual no boiler à noite, eficiência do boiler e radiação solar diária no mês de julho de 2007. Initial and final temperatures in the boiler, speed of the wind, surrounding average temperature at night and day, loss of calorific and percentile energy in the boiler at night, boiler efficiency and solar radiation in July 2007.

\begin{tabular}{|c|c|c|c|c|c|c|c|c|c|}
\hline Data & $\begin{array}{l}\text { T boiler } \\
\text { Início } \\
(6 \mathrm{~h}) \\
{ }^{\circ} \mathrm{C}\end{array}$ & $\begin{array}{c}\text { T boiler } \\
\text { Final } \\
(19 \mathrm{~h} 30) \\
{ }^{\circ} \mathrm{C}\end{array}$ & $\begin{array}{l}\text { Vento } \\
\text { Média } \\
\mathrm{km} \mathrm{h}^{-1}\end{array}$ & $\begin{array}{l}\text { T Ambiente } \\
\text { Média Noite } \\
{ }^{\circ} \mathrm{C}\end{array}$ & $\begin{array}{l}\text { T Ambiente } \\
\text { Média Dia } \\
{ }^{\circ} \mathrm{C}\end{array}$ & $\begin{array}{l}\text { Perda } \\
\text { boiler } \\
\text { Noite } \\
{ }^{\circ} \mathrm{C}\end{array}$ & Perda & $\begin{array}{c}\text { Eficiência } \\
\text { boiler } \\
\%\end{array}$ & $\begin{array}{c}\text { Radiação } \\
\text { Diária }\end{array}$ \\
\hline $22-7-2007$ & 41,80 & 38,10 & 7,63 & 18,32 & 16,25 & & & & \\
\hline $23-7-2007$ & 33,80 & 47,30 & 8,58 & 13,84 & 11,29 & 4,30 & 11,29 & 88,71 & $3.321,43$ \\
\hline 24-7-2007 & 39,90 & 36,70 & 12,03 & 9,10 & 9,48 & 7,40 & 15,64 & 84,36 & $3.458,25$ \\
\hline $25-7-2007$ & 32,40 & 50,60 & 13,49 & 9,91 & 9,57 & 4,30 & 11,72 & 88,28 & $4.552,93$ \\
\hline $26-7-2007$ & 41,30 & 57,20 & 6,35 & 3,09 & 10,16 & 9,30 & 18,38 & 81,62 & $4.621,66$ \\
\hline 27-7-2007 & 47,30 & 61,70 & 5,98 & 6,67 & 14,16 & 9,90 & 17,31 & 82,69 & $4.591,09$ \\
\hline $28-7-2007$ & 51,40 & 61,40 & 8,91 & 9,63 & 15,79 & 10,30 & 16,69 & 83,31 & $4.598,32$ \\
\hline $29-7-2007$ & 50,50 & 56,60 & 19,38 & 8,04 & 13,40 & 10,90 & 17,75 & 82,25 & $4.677,01$ \\
\hline $30-7-2007$ & 47,30 & 38,60 & 13,15 & 10,16 & 15,45 & 9,30 & 16,43 & 83,57 & $3.269,32$ \\
\hline $31-7-2007$ & 33,80 & 57,70 & 7,44 & 11,39 & 17,44 & 4,80 & 12,44 & 87,56 & $4.483,40$ \\
\hline
\end{tabular}


TABELA 2. Temperaturas inicial e final no boiler, velocidade do vento, temperatura média ambiente à noite e de dia, perda de energia calorífica e percentual no boiler à noite, eficiência do boiler e radiação solar diária no mês de agosto de 2007. Initial and final temperatures in the boiler, speed of the wind, surrounding average temperature at night and day, loss of calorific and percentile energy in the boiler at night, boiler efficiency and solar radiation in August 2007.

\begin{tabular}{|c|c|c|c|c|c|c|c|c|c|}
\hline Data & $\begin{array}{l}\text { T boiler } \\
\text { Início } \\
(6 \mathrm{~h}) \\
{ }^{\circ} \mathrm{C}\end{array}$ & $\begin{array}{c}\text { T boiler } \\
\text { Final } \\
(19 \mathrm{~h} 30) \\
{ }^{\circ} \mathrm{C}\end{array}$ & $\begin{array}{l}\text { Vento } \\
\text { Média } \\
\mathrm{km} \mathrm{h}^{-1}\end{array}$ & $\begin{array}{l}\text { T Ambiente } \\
\text { Média Noite } \\
{ }^{\circ} \mathrm{C}\end{array}$ & $\begin{array}{l}\text { T Ambiente } \\
\text { Média Dia } \\
{ }^{\circ} \mathrm{C}\end{array}$ & $\begin{array}{l}\text { Perda } \\
\text { boiler } \\
\text { Noite } \\
{ }^{\circ} \mathrm{C}\end{array}$ & Perda & $\begin{array}{c}\text { Eficiência } \\
\text { boiler } \\
\%\end{array}$ & $\begin{array}{c}\text { Radiação } \\
\text { Diária } \\
\text { Wh m }^{-2}\end{array}$ \\
\hline $1^{\mathrm{o--}} 8-2007$ & 49,30 & 63,60 & 8,76 & 14,61 & 22,34 & 8,40 & 14,56 & 85,44 & $4.339,79$ \\
\hline $2-8-2007$ & 55,00 & 54,70 & 9,18 & 19,75 & 20,48 & 8,60 & 13,52 & 86,48 & $3.147,25$ \\
\hline $3-8-2007$ & 48,00 & 62,80 & 8,15 & 18,78 & 21,47 & 6,70 & 12,25 & 87,75 & $4.354,02$ \\
\hline 4-8-2007 & 53,00 & 45,60 & 7,54 & 12,53 & 19,57 & 9,80 & 15,61 & 84,39 & $3.496,21$ \\
\hline $5-8-2007$ & 39,30 & 57,80 & 7,66 & 9,98 & 18,27 & 6,30 & 13,82 & 86,18 & $4.305,01$ \\
\hline $6-8-2007$ & 49,60 & 55,30 & 15,15 & 16,11 & 21,76 & 8,20 & 14,19 & 85,81 & $3.748,29$ \\
\hline $7-8-2007$ & 47,40 & 58,90 & 8,63 & 15,62 & 14,61 & 7,90 & 14,29 & 85,71 & $4.242,88$ \\
\hline 8-8-2007 & 49,30 & 61,50 & 10,77 & 11,07 & 22,16 & 9,60 & 16,30 & 83,70 & $4.343,28$ \\
\hline $9-8-2007$ & 52,90 & 55,80 & 18,35 & 19,90 & 24,13 & 8,60 & 13,98 & 86,02 & $3.779,29$ \\
\hline $10-8-2007$ & 48,60 & 46,40 & 12,66 & 19,77 & 17,48 & 7,20 & 12,90 & 87,10 & $3.114,25$ \\
\hline $11-8-2007$ & 40,20 & 51,00 & 9,84 & 11,27 & 16,95 & 6,20 & 13,36 & 86,64 & $3.593,17$ \\
\hline $12-8-2007$ & 44,70 & 58,30 & 18,20 & 17,28 & 22,65 & 6,30 & 12,35 & 87,65 & $4.445,58$ \\
\hline $13-8-2007$ & 50,10 & 55,90 & 16,49 & 18,06 & 22,89 & 8,20 & 14,07 & 85,93 & $4.141,25$ \\
\hline $14-8-2007$ & 48,70 & 56,50 & 21,10 & 19,64 & 23,90 & 7,20 & 12,88 & 87,12 & $4.468,09$ \\
\hline $15-8-2007$ & 49,00 & 63,30 & 15,23 & 19,91 & 24,24 & 7,50 & 13,27 & 86,73 & $4.822,41$ \\
\hline $16-8-2007$ & 54,40 & 68,40 & 8,44 & 19,97 & 24,77 & 8,90 & 14,06 & 85,94 & $4.923,60$ \\
\hline $17-8-2007$ & 58,50 & 63,60 & 9,52 & 19,67 & 23,15 & 9,90 & 14,47 & 85,53 & $4.459,30$ \\
\hline $18-8-2007$ & 53,50 & 65,20 & 3,86 & 12,43 & 19,09 & 10,10 & 15,88 & 84,12 & $4.654,06$ \\
\hline $19-8-2007$ & 55,50 & 58,90 & 9,28 & 16,94 & 15,80 & 9,70 & 14,88 & 85,12 & $3.839,51$ \\
\hline 20-8-2007 & 48,70 & 59,60 & 10,64 & 9,15 & 12,33 & 10,20 & 17,32 & 82,68 & $5.246,92$ \\
\hline $21-8-2007$ & 48,90 & 66,10 & 4,42 & 8,39 & 19,44 & 10,70 & 17,95 & 82,05 & $5.201,80$ \\
\hline $22-8-2007$ & 56,60 & 64,60 & 11,95 & 17,62 & 23,82 & 9,50 & 14,37 & 85,63 & $4.826,66$ \\
\hline $23-8-2007$ & 56,20 & 65,50 & 15,41 & 19,43 & 25,19 & 8,40 & 13,00 & 87,00 & $4.945,19$ \\
\hline $24-8-2007$ & 56,20 & 65,30 & 12,76 & 20,54 & 26,36 & 9,30 & 14,20 & 85,80 & $4.837,50$ \\
\hline $25-8-2007$ & 56,50 & 55,90 & 8,45 & 21,79 & 24,25 & 8,80 & 13,48 & 86,52 & $3.441,05$ \\
\hline 26-8-2007 & 49,50 & 41,20 & 10,73 & 20,84 & 23,59 & 6,40 & 11,45 & 88,55 & $2.954,25$ \\
\hline $27-8-2007$ & 35,50 & 29,40 & 8,99 & 9,33 & 12,58 & 5,70 & 13,83 & 86,17 & $2.854,36$ \\
\hline 28-8-2007 & 26,00 & 22,60 & 4,82 & 6,99 & 9,58 & 3,40 & 11,56 & 88,44 & $2.621,36$ \\
\hline 29-8-2007 & 20,80 & 49,50 & 2,35 & 9,78 & 18,27 & 1,80 & 7,96 & 92,04 & $4.561,03$ \\
\hline $30-8-2007$ & 43,40 & 37,30 & 7,24 & 16,03 & 19,56 & 6,10 & 12,32 & 87,68 & $3.475,37$ \\
\hline $31-8-2007$ & 33,70 & 48,00 & 3,55 & 15,81 & 20,09 & 3,60 & 9,65 & 90,35 & $3.559,29$ \\
\hline
\end{tabular}


TABELA 3. Temperaturas inicial e final no boiler, velocidade do vento, temperatura média ambiente à noite e de dia, perda de energia calorífica e percentual no boiler à noite, eficiência do boiler e radiação solar diária no mês de setembro de 2007. Initial and final temperatures in the boiler, speed of the wind, surrounding average temperature at night and day, loss of calorific and percentile energy in the boiler at night, boiler efficiency and solar radiation in September 2007.

\begin{tabular}{|c|c|c|c|c|c|c|c|c|c|}
\hline Data & $\begin{array}{l}\text { T boiler } \\
\text { Início } \\
(6 \mathrm{~h}) \\
{ }^{\circ} \mathrm{C} \\
\end{array}$ & $\begin{array}{c}\mathrm{T} \text { boiler } \\
\text { Final } \\
(19 \mathrm{~h} 30) \\
{ }^{\circ} \mathrm{C} \\
\end{array}$ & $\begin{array}{l}\text { Vento } \\
\text { Média } \\
\mathrm{km} \mathrm{h}^{-1}\end{array}$ & $\begin{array}{l}\text { T Ambiente } \\
\text { Média Noite } \\
{ }^{\circ} \mathrm{C}\end{array}$ & $\begin{array}{l}\text { T Ambiente } \\
\text { Média Dia } \\
{ }^{\circ} \mathrm{C}\end{array}$ & $\begin{array}{l}\text { Perda } \\
\text { boiler } \\
\text { Noite } \\
{ }^{\circ} \mathrm{C} \\
\end{array}$ & Perda & $\begin{array}{c}\text { Eficiência } \\
\text { boiler } \\
\%\end{array}$ & $\begin{array}{c}\text { Radiação } \\
\text { Diária } \\
\text { Wh m }^{-2}\end{array}$ \\
\hline $1^{\circ}-9-2007$ & 42,20 & 61,10 & 3,98 & 16,92 & 25,20 & 5,80 & 12,08 & 87,92 & $4.861,83$ \\
\hline $2-9-2007$ & 53,30 & 68,90 & 6,47 & 20,89 & 27,35 & 7,80 & 12,77 & 87,23 & $5.375,04$ \\
\hline $3-9-2007$ & 59,30 & 69,60 & 11,97 & 22,13 & 27,11 & 9,60 & 13,93 & 86,07 & $5.481,98$ \\
\hline 4-9-2007 & 59,70 & 67,30 & 18,40 & 22,38 & 27,09 & 9,90 & 14,22 & 85,78 & $5.355,03$ \\
\hline $5-9-2007$ & 58,20 & 67,40 & 12,87 & 22,64 & 27,21 & 9,10 & 13,52 & 86,48 & $5.265,92$ \\
\hline $6-9-2007$ & 58,30 & 71,30 & 9,89 & 22,52 & 27,14 & 9,10 & 13,50 & 86,50 & $5.676,08$ \\
\hline $7-9-2007$ & 61,10 & 69,60 & 9,60 & 22,56 & 27,21 & 10,20 & 14,31 & 85,69 & $5.477,40$ \\
\hline $8-9-2007$ & 60,00 & 70,10 & 14,56 & 21,79 & 27,98 & 9,60 & 13,79 & 86,21 & $5.719,30$ \\
\hline $9-9-2007$ & 60,30 & 70,60 & 14,20 & 22,66 & 27,82 & 9,80 & 13,98 & 86,02 & $5.699,42$ \\
\hline $10-9-2007$ & 60,60 & 70,10 & 14,15 & 22,21 & 27,29 & 10,00 & 14,16 & 85,84 & $5.787,02$ \\
\hline $11-9-2007$ & 60,20 & 67,10 & 15,63 & 22,43 & 26,71 & 9,90 & 14,12 & 85,88 & $5.449,35$ \\
\hline $12-9-2007$ & 58,00 & 57,00 & 14,39 & 22,36 & 24,25 & 9,10 & 13,56 & 86,44 & $3.477,14$ \\
\hline $13-9-2007$ & 50,20 & 67,10 & 15,04 & 22,14 & 26,69 & 6,80 & 11,93 & 88,07 & $5.842,53$ \\
\hline $14-9-2007$ & 57,90 & 35,10 & 12,42 & 22,36 & 25,36 & 9,20 & 13,71 & 86,29 & $2.951,36$ \\
\hline $15-9-2007$ & 32,60 & 31,00 & 9,70 & 24,28 & 26,28 & 2,50 & 7,12 & 92,88 & $3.332,98$ \\
\hline $16-9-2007$ & 29,50 & 57,60 & 4,94 & 19,68 & 26,26 & 1,50 & 4,84 & 95,16 & $4.830,90$ \\
\hline
\end{tabular}

Na Figura 2, estão plotadas a temperatura final da água no boiler (às $19 \mathrm{~h} 30$ ), em ${ }^{\circ} \mathrm{C}$, e a velocidade média do vento, em $\mathrm{km} \mathrm{h}^{-1}$, no período de 22-7 a 16-9 de 2007. Conclui-se que não há relação entre a temperatura final da água no boiler e a velocidade do vento. Isso ocorre porque, apesar de a placa estar exposta, o boiler está sob o telhado, não sofrendo ação direta das intempéries.

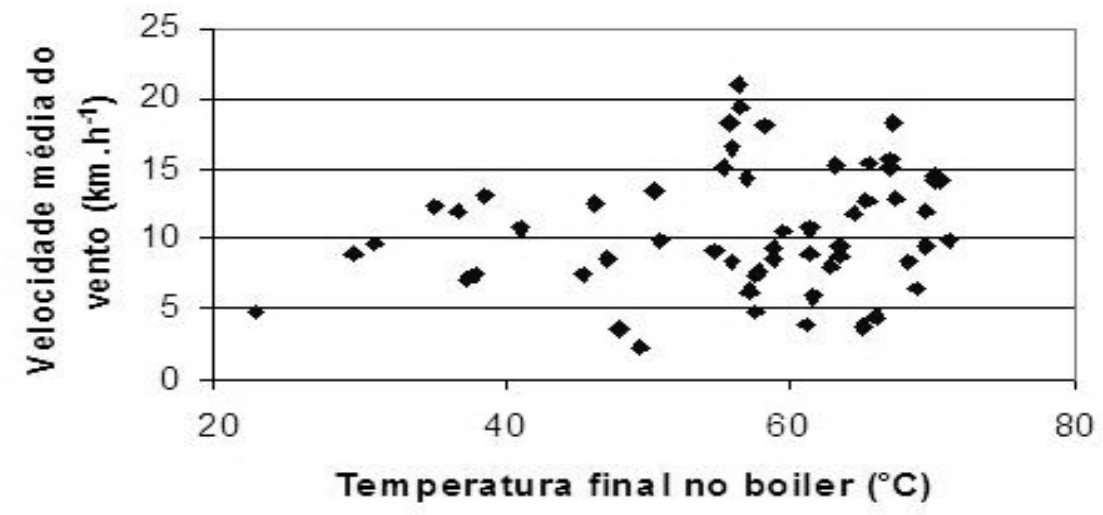

FIGURA 2. Relação entre a temperatura final da água no boiler e a velocidade do vento, no período de inverno de 2007. Relation between the boiler's water temperature and the wind's speed, in 2007's winter period.

Na Figura 3, estão plotados os valores de temperatura da água na entrada e na saída da placa, no boiler e a velocidade do vento, do dia 28 de julho de 2007, às 17h32, até o dia 29 de julho de 2007, às $17 \mathrm{~h} 22$, quando ocorreu a maior média de velocidade do vento, $19,38 \mathrm{~km} \mathrm{~h}^{-1}$. No eixo das abscissas, é apresentada a hora do dia, enquanto no eixo das ordenadas, são apresentados, em valores absolutos, as temperaturas e a velocidade do vento. No período de maior velocidade do vento (em torno de $11 \mathrm{~h}$ ), são altas as temperaturas de entrada e de saída da placa e também da água 
no boiler, confirmando que não há interferência do vento na temperatura da água. Observa-se que o declínio da temperatura no boiler durante à noite corresponde à perda térmica, e o posterior aumento após as $11 \mathrm{~h}$, em virtude do aquecimento da água na placa, o que permite o acréscimo de temperatura da água no boiler.

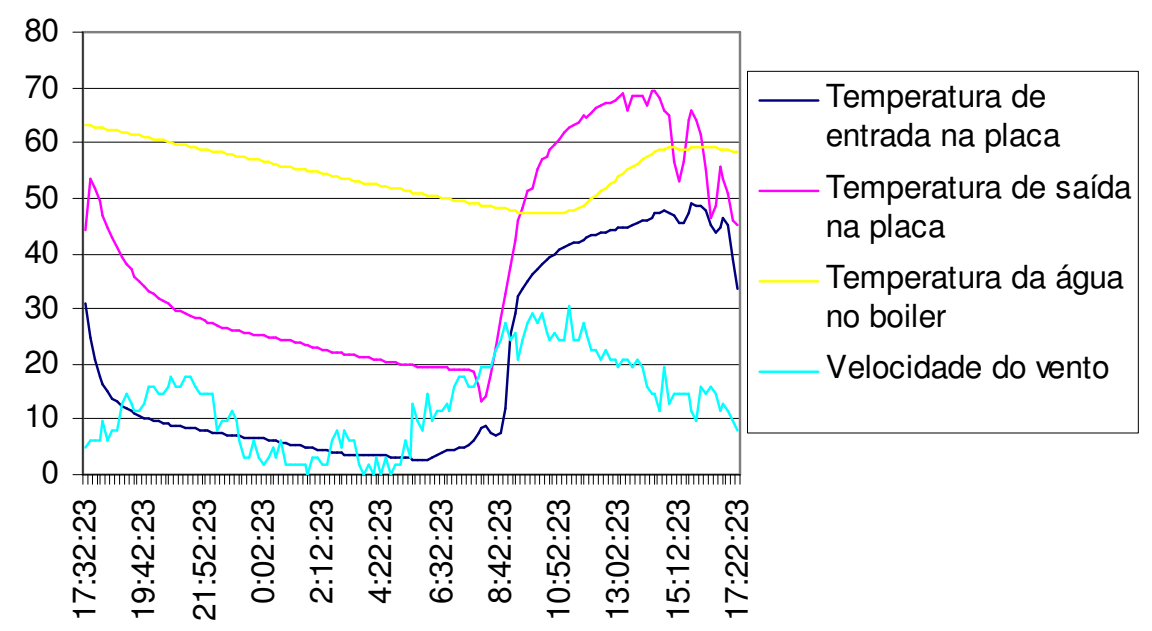

FIGURA 3. Relação entre as temperaturas da água na entrada e saída da placa, da água do boiler e da velocidade do vento, no período de 24 horas. Relation between the temperatures of the water in the entrance and exit of the plate, the boiler's water and the wind's speed, in a 24 hour period.

Na Figura 4, estão plotadas a temperatura ambiente média e a perda térmica no boiler durante a noite. Verifica-se que não há relação entre a perda térmica de energia do boiler com a temperatura ambiente média da noite.

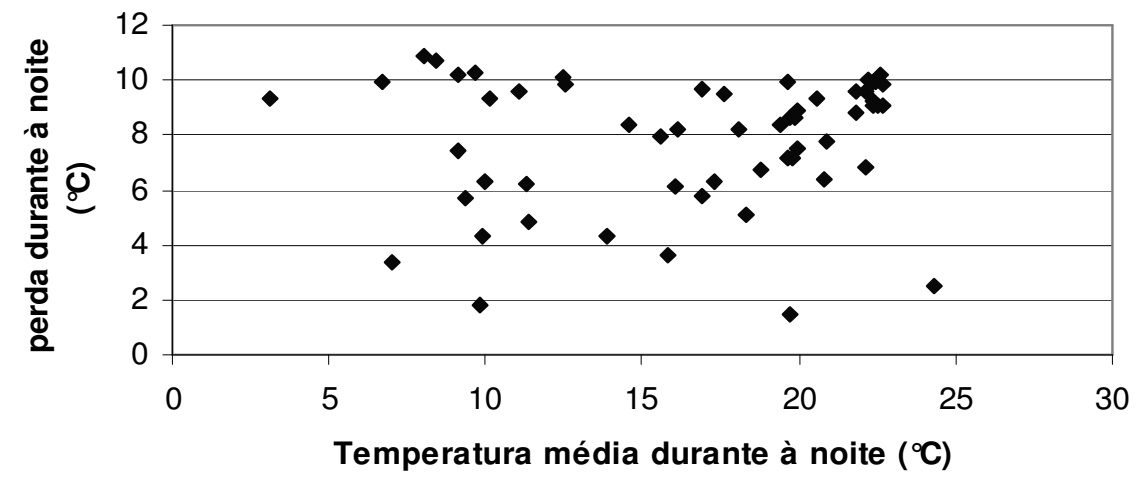

FIGURA 4. Temperatura ambiente média durante a noite e perda da energia térmica no boiler, durante a noite. Average ambient temperature at night and loss of the thermal energy in the boiler, at night. boiler.

Na Figura 5, são apresentadas a radiação diária na placa e a temperatura final da água no

A maioria das medidas obtidas possui relação entre a radiação diária e a temperatura final da água no boiler. Portanto, verifica-se que o principal fator para o aquecimento da água no sistema estudado é a radiação solar, confirmando as afirmações de BRAZIL (2006). 


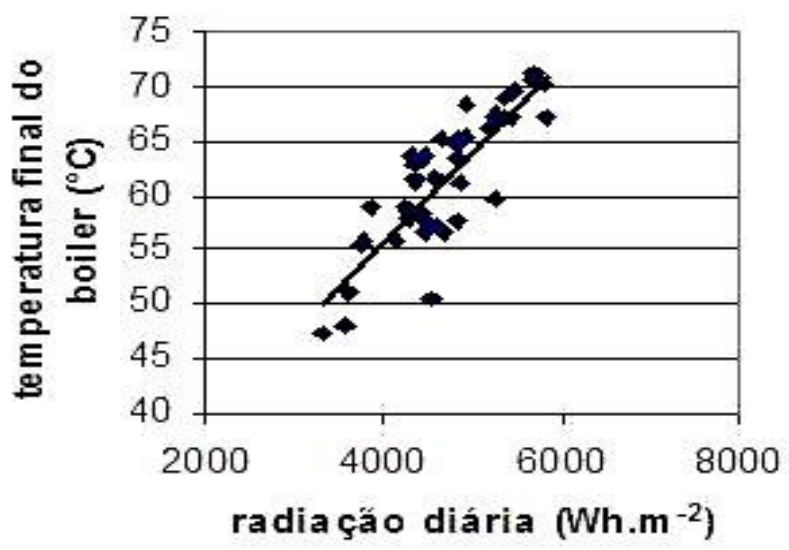

$y=0,0083 x+22,489$
$R^{2}=0,7383$

FIGURA 5. Temperatura final da água no boiler em relação à radiação total diária no inverno. Final temperature of the water in the boiler related to the daily total radiation in the winter.

\section{CONCLUSÕES}

Conclui-se que há viabilidade técnica na instalação do equipamento de aquecimento de água por energia solar na região oeste do Paraná para o aquecimento de água residencial rural. O equipamento permite alcançar temperaturas mínimas da água para banho em número de dias suficientes, para que o aquecimento elétrico seja apenas complementar e utilizado esporadicamente.

Mantidas as especificações do fabricante, o equipamento funciona de maneira satisfatória e a manutenção do equipamento de aquecimento de água esteve restrita à limpeza da placa, que pode ser realizada pelo próprio usuário. Não houve desgaste aparente dos materiais expostos às intempéries no período estudado.

\section{REFERÊNCIAS}

BRAZIL, O.A.V. Regulação e apropriação de energia térmica solar pela população de baixa renda no Brasil. 2006. 121 f. Dissertação (Mestrado em Regulação da Indústria de Energia) Universidade Salvador, Salvador, 2006.

CHANG, J.M.; LEU, J.S.; SHEN, M.C.; HUANG, B.J. A proposed modified efficiency for thermosyphon solar heating systems. Solar Energy, Great Britain, v.76, n.6, p.693-701, 2004.

CREDER, H. Instalações hidráulicas e sanitárias. Rio de Janeiro: Livros Técnicos e Científicos, 2006. 423 p.

DHARUMAN, C.; ARAKERI, J.H.; SRINIVASAN, K. Performance evaluation of an integrated solar water heater as an option for building energy conservation. Energy and Buildings, United Kingdom, v.38, n.3, p.214-219, 2006.

KHALIFA, A-J.N. Thermal performance of locally made flat plate solar collectors used as part of a domestic hot water system. Energy Conversion \& Management, United Kingdom, v.40, n.17, p.1.825-1.833, 1999.

KOLLING, E.M.; SOUZA, S.N.M.; RICIERI. R.; SAMPAIO, S.C.; DALLACORT. R. Análise operacional de um sistema fotovoltaico de bombeamento de água. Engenharia Agrícola, Jaboticabal, v.24, n.3, p.527-535, 2004.

KULKARNI, G.N.; KEDARE, S.B.; BANDYOPADHYAY, S. Determination of design space and optimization of solar water heating systems. Solar Energy, Great Britain, v.81, n.10, p.958-968, 2007.

MADHLOPA, A.; MGAWI, R.; TAULO, J. Experimental study of temperature stratification in a integrated colletor-storage solar water heater with two horizontal tanks. Solar Energy, Great Britain, v.80, n.8, p.989-1.002, 2006. 